\title{
Remark on the equisingularity of families of affine plane curves
}

\author{
by HÀ Huy Vui (Hanoi) and Phạm Tien Son (Dalat)
}

\begin{abstract}
We give some criteria for the equisingularity of families of affine plane curves.

1. Introduction. Let $f_{\alpha}: \mathbb{C}^{2} \rightarrow \mathbb{C}$ be a family of polynomials whose coefficients are polynomial functions of $\alpha \in \mathbb{C}^{n}$. Consider the family of affine curves $V_{\alpha}:=\left\{(x, y) \in \mathbb{C}^{2} \mid f_{\alpha}(x, y)=0\right\}, \alpha \in \mathbb{C}^{n}$. The aim of this paper is to give certain necessary and sufficient conditions for the family $\left\{V_{\alpha}\right\}_{\alpha \in \mathbb{C}^{n}}$ to be equisingular. These conditions read as follows: apart from the requirement that the curves $V_{\alpha}$ satisfy Whitney's conditions at each common critical point (or equivalently, $\mu_{a}\left(V_{\alpha}\right)=$ const at such a point $a$, where $\mu_{a}\left(V_{\alpha}\right)$ denotes the Milnor number of the curve $V_{\alpha}$ at $\left.a\right)$ they need to have good behavior at infinity (i.e., in a sense, $V_{\alpha}$ satisfy the so-called Whitney's affine conditions at infinity).

We now suppose that the affine curves $V_{\alpha}, \alpha \in \mathbb{C}^{n}$, all have the same critical points, say $a_{i}=\left(x_{i}, y_{i}\right) \in \mathbb{C}^{2}, i=1, \ldots, s$.

1.1. Definition. The family of affine curves $V_{\alpha}$ is said to be equisingular if for all $\alpha^{0} \in \mathbb{C}^{n}$ there exist a neighborhood $U_{\alpha^{0}}$ of $\alpha^{0}$ and a diffeomorphism $h$ such that $h\left(a_{i}, \alpha\right)=\left(a_{i}, \alpha\right), i=1, \ldots, s$, and the diagram

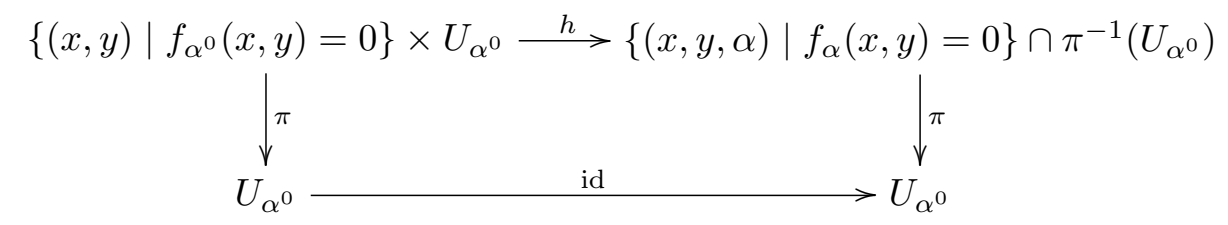

1991 Mathematics Subject Classification: 32S55, 57M25, 57Q45.

Key words and phrases: affine curves, singularity, equisingularity, Milnor number, Euler characteristic, Whitney's conditions.

Supported in part by the National Basic Research Program in Natural Sciences, Vietnam. The second author would like to thank "l'Association d'Aubonne Culture et Education France-Vietnam" for its financial support. 
is commutative, where $\pi$ is the second projection. Let $\Gamma:=\{(x, y, \alpha) \in$ $\left.\mathbb{C}^{2} \times \mathbb{C}^{n} \mid f_{\alpha}(x, y)=0\right\}$.

1.2. Definition. The family of affine curves $V_{\alpha}$ is said to be good at infinity if for each $\alpha^{0} \in \mathbb{C}^{n}$, there exist $c>0$ and a neighborhood $U_{\alpha^{0}}$ of $\alpha^{0}$ such that the tangent hyperplane $T_{(u, v, \beta)}(\Gamma \cap\{\alpha=\beta\})$ is transverse within the plane $\{\alpha=\beta\} \subset \mathbb{C}^{2} \times \mathbb{C}^{n}$ to the line $\{x=u, \alpha=\beta\}$ for all $(u, v, \beta) \in \Gamma$, $\beta \in U_{\alpha^{0}},|u| \geq c$.

1.3. REMARK. (a) Although the above definition is based on a specific (and explicit) choice of the line $\{x=u, \alpha=\beta\}$, it is easily seen that we can choose $\left\{l_{1} x+l_{2} y=l_{1} u+l_{2} v, \alpha=\beta\right\},\left(l_{1}: l_{2}\right) \in \mathbb{C P}^{1}$, instead.

(b) By definition, the family $\left\{V_{\alpha}\right\}_{\alpha \in \mathbb{C}^{n}}$ is good at infinity if and only if there exist $c>0$ and a neighborhood $U_{\alpha^{0}}$ of $\alpha^{0}$ such that

$$
\frac{\partial f_{\alpha}}{\partial y} \neq 0 \quad \text { for all } \alpha \in U_{\alpha^{0}}, x, y \in \mathbb{C} \text { with }|x| \geq c, f_{\alpha}(x, y)=0 .
$$

(c) The following assumptions will be made throughout this paper:

- the curves $\left\{f_{\alpha}(x, y)=0\right\}$ are all reduced;

- $d:=\operatorname{deg}\left(f_{\alpha}\right)=\operatorname{deg}_{y}\left(f_{\alpha}\right)$.

The second assumption implies that the restriction map

$$
\left.l\right|_{V_{\alpha}}: V_{\alpha} \rightarrow \mathbb{C}, \quad(x, y) \mapsto x,
$$

is proper. Let $\delta(x, \alpha):=\operatorname{disc}_{y}\left(f_{\alpha}(x, y)\right)$ be the discriminant of $f_{\alpha}$ with respect to $y$. Then we can write

$$
\delta(x, \alpha)=q_{k}(\alpha) x^{k}+q_{k-1}(\alpha) x^{k-1}+\ldots+q_{0}(\alpha), \quad q_{k} \not \equiv 0,
$$

where $q_{i}(\alpha), i=0, \ldots, k$, are polynomials of $\alpha$. Therefore, by the properties of resultants, the family $\left\{V_{\alpha}\right\}_{\alpha \in \mathbb{C}^{n}}$ is good at infinity if and only if $q_{k}(\alpha)=$ const $\neq 0$.

2. The main result of this paper is the following theorem.

2.1. Theorem. Suppose that the affine curves $V_{\alpha}, \alpha \in \mathbb{C}^{n}$, have the same critical points, say $a_{i}=\left(x_{i}, y_{i}\right) \in \mathbb{C}^{2}, i=1, \ldots, s$. Then the following two conditions are equivalent:

(a) the family $\left\{V_{\alpha}\right\}_{\alpha \in \mathbb{C}^{n}}$ is equisingular;

(b) $\mu_{a_{i}}\left(V_{\alpha}\right)=$ const, $i=1, \ldots, s$; and the family $\left\{V_{\alpha}\right\}_{\alpha \in \mathbb{C}^{n}}$ is good at infinity.

For the proof we need the below lemma. 
2.2. Lemma ([4]). Let there be given a polynomial $F$ of two complex variables and the map

$$
l: \mathbb{C}^{2} \rightarrow \mathbb{C}, \quad(x, y) \mapsto x,
$$

such that the restriction map $l_{V}, V:=F^{-1}(0)$, is proper. Moreover, suppose that the curve $V$ is reduced. Then

$$
\chi\left(F^{-1}(0)\right)=d-\operatorname{deg} \operatorname{disc}_{y} F(x, y),
$$

where $d:=\operatorname{deg}_{y}(F)$ and $\chi\left(F^{-1}(0)\right)$ is the Euler characteristic of $F^{-1}(0)$.

Proof of Theorem 2.1. (a) $\Rightarrow(\mathrm{b})$ is easy. Indeed, the Milnor number is a topological invariant for isolated curve singularities [5]; hence,

$$
\mu_{a_{i}}\left(V_{\alpha}\right)=\text { const }, \quad i=1, \ldots, s .
$$

Moreover, by the definition of equisingularity, $\chi\left(V_{\alpha}\right)=$ const. Therefore, according to Lemma $2.2, q_{k}(\alpha)=$ const. So the family $\left\{V_{\alpha}\right\}_{\alpha \in \mathbb{C}^{n}}$ is good at infinity by Remark 1.3(c).

$(\mathrm{b}) \Rightarrow(\mathrm{a})$. We denote by grad $f$ the vector $\operatorname{grad} f:=\overline{(\partial f / \partial x, \partial f / \partial y)}$, so the chain rule may be expressed by the inner product $\partial f / \partial v=\langle v$, grad $f\rangle$. Assume that $\alpha^{0} \in \mathbb{C}^{n}$. Since $\mu_{a_{i}}\left(V_{\alpha}\right)=$ const, $i=1, \ldots, s$, there exists a neighborhood $U_{\alpha^{0}}$ of $\alpha^{0}$ such that the family $\left\{V_{\alpha}\right\}_{\alpha \in \mathbb{C}^{n}}$ satisfies Whitney's conditions along $\left\{a_{i}\right\} \times U_{\alpha^{0}}$ at $a_{i}, i=1, \ldots, s$ (see [6], [2]). Thus there exist closed balls $D_{i}$ small enough centered at $a_{i}$ such that $D_{i} \cap D_{j}=\emptyset(i \neq j)$ and there exist integrable vector fields $\bar{\xi}^{i j}(x, y, \alpha), \bar{\eta}^{i j}(x, y, \alpha), i=1, \ldots, s$, $j=1, \ldots, n$, nowhere zero on the set

$$
\Gamma_{\alpha^{0}}^{i}:=\left\{(x, y, \alpha) \in D_{i} \times U_{\alpha^{0}} \mid f_{\alpha}(x, y)=0\right\}
$$

such that the following equations are satisfied:

$$
\begin{array}{r}
\left\langle\bar{\xi}^{i j}(x, y, \alpha), \operatorname{grad} f_{\alpha}(x, y)\right\rangle+\frac{\partial f_{\alpha}}{\partial \alpha_{j}}(x, y)=0, \\
\left\langle\bar{\eta}^{i j}(x, y, \alpha), \operatorname{grad} f_{\alpha}(x, y)\right\rangle+\sqrt{-1} \frac{\partial f_{\alpha}}{\partial \alpha_{j}}(x, y)=0,
\end{array}
$$

for all $(x, y, \alpha) \in\left\{(x, y, \alpha) \in \partial D_{i} \times U_{\alpha^{0}} \mid f_{\alpha}(x, y)=0\right\}$. Moreover, by the same method as in [4], integrating the above vector fields, we can get the diffeomorphisms $h_{i}, i=1, \ldots, s$, such that the diagrams

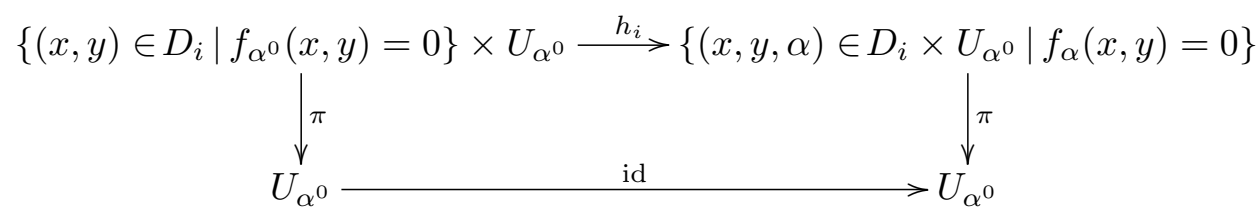

are commutative and $h_{i}\left(a_{i}, \alpha\right)=\left(a_{i}, \alpha\right)$. 
Further, by Remark 1.3(b), there exist $c>0$ and a neighborhood of $\alpha^{0}$, which we may assume to be precisely $U_{\alpha^{0}}$, such that

$$
\frac{\partial f_{\alpha}}{\partial y} \neq 0 \quad \text { for all }(x, y, \alpha) \in \Gamma_{\alpha^{0}} \cup \Omega,
$$

where

$$
\Gamma_{\alpha^{0}}:=\left\{(x, y, \alpha) \in \mathbb{C}^{2} \times \mathbb{C}^{n}\left|f_{\alpha}(x, y)=0, \alpha \in U_{\alpha^{0}},\right| x \mid \geq c\right\}
$$

and $\Omega$ is an open neighborhood of the set

$$
\left\{(x, y, \alpha) \in \mathbb{C}^{2} \times \mathbb{C}^{n}\left|f_{\alpha}(x, y)=0, \alpha \in U_{\alpha^{0}},\right| x \mid=c\right\} .
$$

On the other hand, one has

(2) $\operatorname{grad} f_{\alpha} \neq 0 \quad$ for all $(x, y, \alpha) \in\left(\mathbb{C}^{2} \backslash \bigcup_{i=1}^{s} \stackrel{\circ}{D}_{i}\right) \times U_{\alpha^{0}}$

$$
\text { with } f_{\alpha}(x, y)=0,|x| \leq c \text {. }
$$

From (1) and (2) we conclude that there exist smooth vector fields

$$
\begin{aligned}
\xi^{j}(x, y, \alpha) & =\left(\xi_{1}^{j}(x, y, \alpha), \xi_{2}^{j}(x, y, \alpha)\right), \\
\eta^{j}(x, y, \alpha) & =\left(\eta_{1}^{j}(x, y, \alpha), \eta_{2}^{j}(x, y, \alpha)\right), \quad j=1, \ldots, n,
\end{aligned}
$$

such that

$$
\begin{array}{r}
\left\langle\xi^{j}(x, y, \alpha), \operatorname{grad} f_{\alpha}(x, y)\right\rangle+\frac{\partial f_{\alpha}}{\partial \alpha_{j}}(x, y)=0, \\
\left\langle\eta^{j}(x, y, \alpha), \operatorname{grad} f_{\alpha}(x, y)\right\rangle+\sqrt{-1} \frac{\partial f_{\alpha}}{\partial \alpha_{j}}(x, y)=0,
\end{array}
$$

on the set $X:=\left\{(x, y, \alpha) \in \mathbb{C}^{2} \times \mathbb{C}^{n} \mid f_{\alpha}(x, y)=0, \alpha \in U_{\alpha^{0}}\right\}$, and

$$
\xi_{1}^{j}(x, y, \alpha)=0, \quad \eta_{1}^{j}(x, y, \alpha)=0,
$$

for all $(x, y, \alpha) \in \Gamma_{\alpha^{0}} \cup \Omega$.

Furthermore, the restrictions of $\xi^{j}$ (resp., $\eta^{j}$ ), $j=1, \ldots, n$, on $\Gamma_{\alpha^{0}}^{i}$, $i=1, \ldots, s$, are precisely $\bar{\xi}^{i j}$ (resp., $\bar{\eta}^{i j}$ ). (We can construct such vector fields locally and then extend them over $X$ by a smooth partition of unity.) The resulting vector fields on $X$ are the ones we are looking for. Using them, we may follow again the method of [4] to obtain the global diffeomorphism $h$ such that the diagram

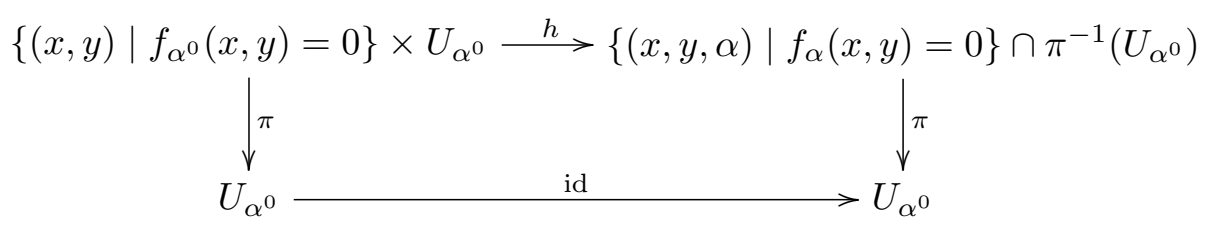

is commutative and $\left.h\right|_{D_{i} \times U_{\alpha^{0}}}=h_{i}, i=1, \ldots, s$. The theorem is proved. 
2.3. REMARK. By definition and the above theorem, it is reasonable to

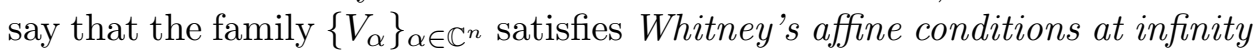
provided it is good at infinity.

3. In $\mathbb{C P}^{2}$ we consider the family of curves

$$
\bar{V}_{\alpha}:=\left\{(x: y: z) \in \mathbb{C P}^{2} \mid z^{d} f_{\alpha}(x / z, y / z)=0\right\}, \quad \alpha \in \mathbb{C}^{n} .
$$

Clearly, $\bar{V}_{\alpha}$ is the compactification of $V_{\alpha}$. From now on we make the assumption that $B:=\bar{V}_{\alpha} \cap\{z=0\} \subset \mathbb{C P}^{2}$ is a finite set, say $\left\{b_{1}, \ldots, b_{m}\right\}$, i.e., the degree $d$ homogeneous parts of $f_{\alpha}$ are independent of $\alpha$.

3.1. Lemma. Suppose that the Milnor numbers of $V_{\alpha}$ are independent of $\alpha$, i.e., $\sum_{a \in V_{\alpha}} \mu_{a}\left(V_{\alpha}\right)=$ const. Then

$$
\chi\left(V_{\alpha}\right)-\chi\left(V_{\alpha^{0}}\right)=\sum_{j=1}^{m}\left[\mu_{b_{j}}\left(\bar{V}_{\alpha}\right)-\mu_{b_{j}}\left(\bar{V}_{\alpha^{0}}\right)\right] .
$$

Proof. According to [3],

$$
\begin{aligned}
\chi\left(V_{\alpha}\right) & =\chi\left(\bar{V}_{\alpha}\right)-m \\
& =2-(d-1)(d-2)+\sum_{a \in V_{\alpha}} \mu_{a}\left(V_{\alpha}\right)+\sum_{j=1}^{m} \mu_{b_{j}}\left(\bar{V}_{\alpha}\right)-m,
\end{aligned}
$$

which completes the proof.

From Theorem 2.1, we obtain the following corollary.

3.2. Corollary. Under the hypotheses of Theorem 2.1, (a), hence (b), is equivalent to

(c) $\mu_{a_{i}}\left(V_{\alpha}\right)=$ const, $i=1, \ldots, s$, and $\mu_{b_{j}}\left(\bar{V}_{\alpha}\right)=$ const, $j=1, \ldots, m$.

Proof. We need only prove (b) $\Leftrightarrow(\mathrm{c})$. From Lemma 2.2 and Remark 1.3(c), the family $\left\{V_{\alpha}\right\}_{\alpha \in \mathbb{C}^{n}}$ is good at infinity if and only if $\chi\left(V_{\alpha}\right)=$ const. But, by Lemma 3.1, this is equivalent to

$$
\sum_{j=1}^{m} \mu_{b_{j}}\left(\bar{V}_{\alpha}\right)=\text { const }
$$

or equivalently (using the semicontinuity of the Milnor number),

$$
\mu_{b_{j}}\left(\bar{V}_{\alpha}\right)=\text { const }, \quad j=1, \ldots, m \text {. }
$$

3.3. Remark. According to [6], [1] and [2], $\mu_{b_{j}}\left(\bar{V}_{\alpha}\right)=$ const, $j=$ $1, \ldots, m$, if and only if the family $\left\{\bar{V}_{\alpha}\right\}_{\alpha \in \mathbb{C}^{n}}$ satisfies Whitney's conditions along $\left\{b_{j}\right\} \times \mathbb{C}^{n}$ at $b_{j}$. In the case of a family of affine plane curves, the equisingularity is therefore equivalent to Whitney's conditions at each common singular point of the curves (including such singular points at infinity). 


\section{References}

[1] J. Briançon et J.-P. Speder, Les conditions de Whitney impliquent " $\mu$ * constant", Ann. Inst. Fourier (Grenoble) 26 (2) (1976), 153-163.

[2] A. Dimca, Singularities and Topology of Hypersurfaces, Universitex, Springer, New York, 1992.

[3] Hà Huy Vui et Lê Dũng Tráng, Sur la topologie des polynômes complexes, Acta Math. Vietnam. 9 (1984), 21-32.

[4] Hà Huy Vui and Phạm Tien Son, Topology of families of affine plane curves, Institute of Mathematics, Hanoi, Vietnam, preprint 1, 1997.

[5] Lê Dũng Tráng, Topologie des singularités des hypersurfaces complexes, Singularités à Cargèse, Astérisque 7-8 (1973), 171-182.

[6] B. Teissier, Cycles évanescents, sections planes et conditions de Whitney, ibid., 285-362.

Institute of Mathematics

P.O. Box 631

Bo-Ho, Hanoi, Vietnam
Department of Mathematics

Dalat University

Dalat, Vietnam

Reçu par la Rédaction le 28.6.1997 